\title{
Sawtooth behaviour in highly elongated TCV plasmas
}

H. Reimerdes, I. Furno, F. Hoffmann, An Martynov, A. Pochelon and O. Sauter

Accepted for publication in Plasma Phys. \& Contr. Fusion 



\title{
Sawtooth behaviour in highly elongated TCV plasmas
}

\author{
H Reimerdesł, I Furno, F Hofmann, An Martynov, A Pochelon \\ and $O$ Sauter
}

Ecole Polytechnique Fédérale de Lausanne (EPFL), Centre de Recherches en Physique des Plasmas, Association EURATOM - Confédération Suisse, CH-1015 Lausanne, Switzerland

E-mail: hr2012@columbia.edu

\begin{abstract}
The effect of plasma elongation $\kappa$ on the sawtooth behaviour is studied in ohmically heated TCV plasmas up to values of $\kappa=2.8$. Above a certain value of $\kappa$, which varies from 2.2 to 2.6 and depends on the current profile, the sawtooth oscillations disappear completely. The sawteeth are then replaced by continuous oscillations with frequencies in the range from 5 to $20 \mathrm{kHz}$, which are resonant at the $q=1$ surface and seem to prevent a peaking of the temperature and pressure profiles in the plasma core. It is suggested that the transition is a consequence of an ideal internal kink mode becoming unstable, even with a flat pressure profile inside the $q=1$ surface, if $\kappa$ is sufficiently high.
\end{abstract}

Accepted for publication in Plasma Phys. Control. Fusion

PACS numbers: 52.30.Cv, 52.35.Py

\section{Introduction}

The shaping of the poloidal plasma cross section has a profound effect on both MHD stability and on energy confinement. Elongated plasmas in particular, have several advantageous properties. The higher current carrying capacity leads to increased beta limits [1] and better confinement, as reflected in the tokamak confinement scaling laws [2]. Another consequence of an increase in plasma elongation is an increase in the † Present address: Columbia University, New York, NY 10027, USA 
sawtooth inversion radius $\rho_{\mathrm{inv}}$ at constant edge safety factor $q_{a}$ [3]. The often assumed correspondence of large sawtooth inversion radius and large sawtooth crash amplitude is a concern for inductive scenarios in a future fusion reactor, since large sawtooth crashes are more likely to provide the seed islands which trigger neoclassical tearing modes, causing severe performance degradation [4]. Previous analysis of sawteeth in the TCV tokamak, however, shows that the sawtooth inversion radius is not necessarily a good indicator of the sawtooth crash amplitude [5, 6]. A systematic shape scan up to an elongation of 2.1, but at constant inversion radius, has revealed a continuous decrease of sawtooth period and sawtooth amplitude with increasing elongation. This decrease in the sawtooth amplitude has been linked to the predicted decrease of the central pressure limit of the ideal internal kink mode. A strong dependence of sawtooth behaviour on plasma shape has also been observed in the DIII-D tokamak [7].

The TCV tokamak (major radius $R=0.88 \mathrm{~m}$, minor radius $a<0.25 \mathrm{~m}$, magnetic field on axis $B_{0}<1.54 \mathrm{~T}$ ), with its vacuum vessel elongation of 2.9 , is a unique device for experimental investigations of the effects of plasma shaping. In TCV, extremely elongated plasmas with an elongation of up to $\kappa=2.8$ have been created [8]. In this article, the behaviour of sawteeth at high elongation is investigated and the change in MHD activity, related to an abrupt disappearance of sawteeth at high elongation, is analysed in detail.

\section{Experimental observations}

In TCV, sawteeth are studied in ohmically heated L-mode discharges with elongations up to $\kappa=2.8$. In these discharges $\kappa$ and the plasma current $I_{p}$ are carefully ramped up simultaneously in order to avoid vertical displacement events and kink modes which impose lower and upper current limits, respectively [8, 9]. The current limits translate into limits to $q_{a}$. Figure 1(a) shows the time evolution of $\kappa$ and $I_{p}$ in a typical high elongation discharge (\#19405) which has a maximum elongation of $\kappa_{\max }=2.65$ with $q_{a} \sim 3.7$.

\subsection{Sawteeth period and inversion radius}

The sawtooth period $\tau_{\mathrm{ST}}$, which is obtained from a soft x-ray signal measured along a central chord, decreases with increasing $\kappa$, figure 1(b). At the highest elongations, 

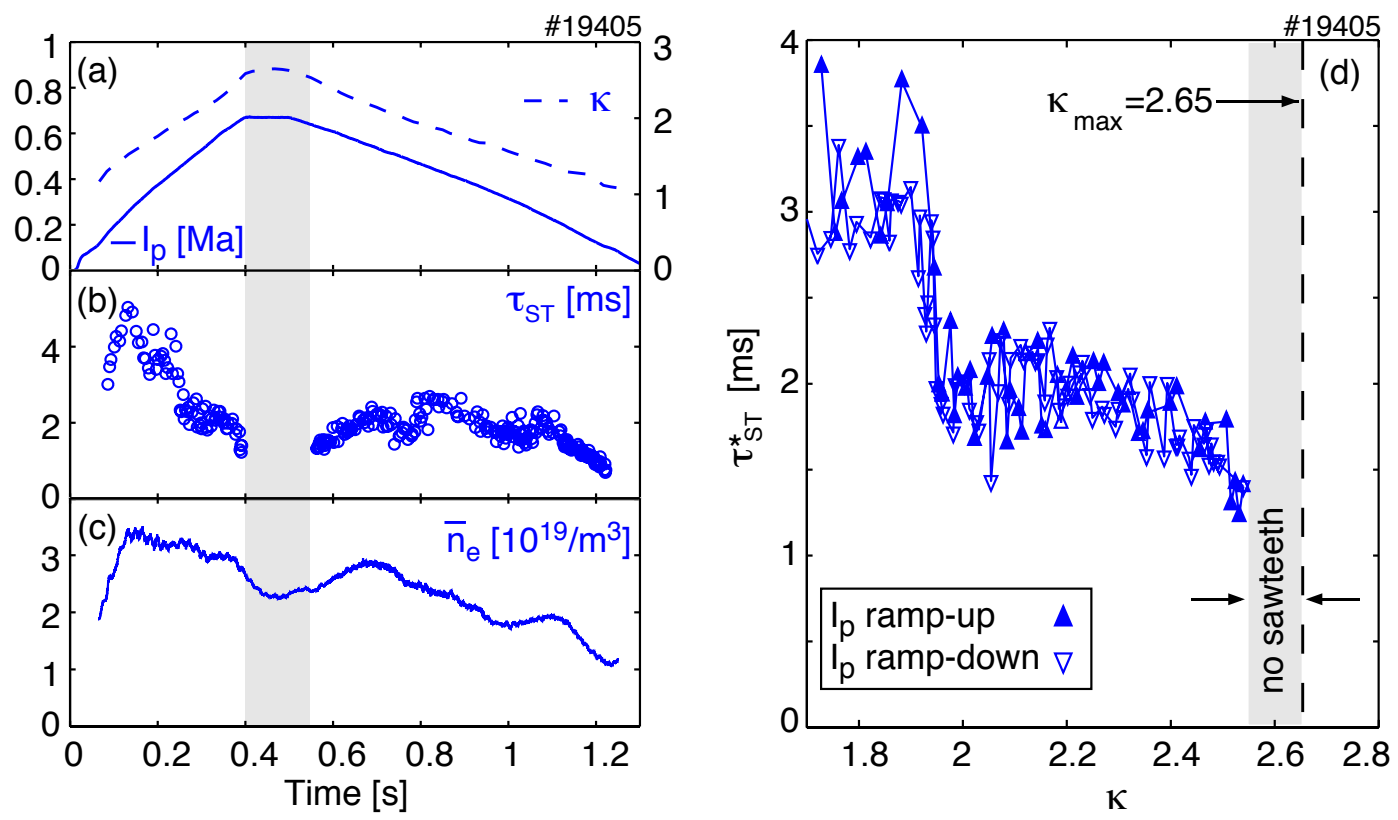

Figure 1. (a): Evolution of the plasma current $I_{p}$ and elongation $\kappa$ in discharge \#19405. (b): The sawtooth period $\tau_{\mathrm{ST}}$ decreases during the ramp-up of $I_{p}$ and $\kappa$. Sawteeth disappear completely from $t=0.40$ to $0.55 \mathrm{~s}$ (shaded). (c): The line averaged density $\bar{n}_{\mathrm{e}}$ varies during the discharge. (d): Normalizing the sawtooth period on the density, $\tau_{\mathrm{ST}}^{*}=\tau_{\mathrm{ST}} \cdot \bar{n}_{\mathrm{e}} / \bar{n}_{\mathrm{e}, \kappa_{\max }}$, removes its dependence on the $I_{p}$ ramp direction.

here for $\kappa>2.53$, sawteeth disappear completely. The value of the sawtooth period in the current ramp-up differs from the value at the same elongation in the rampdown. This hysteresis is attributed to the dependence of the sawtooth period on the electron density $n_{\mathrm{e}}$, which was not kept constant throughout the discharge, figure 1(c). It has been found on several tokamaks that the sawtooth period is proportional to the density, e.g. [10]. The proportionality, $\tau_{\mathrm{ST}} \propto n_{\mathrm{e}}$ is also confirmed in TCV for different elongations $(\kappa=1.6$ and 2.2$)$, at similar measured inversion radii $\left(\rho_{\mathrm{inv}} \approx 0.45\right)$ with densities ranging from 2 to $7 \times 10^{19} \mathrm{~m}^{-3}$. A normalisation of the sawtooth period on the (line averaged) electron density, $\tau_{\mathrm{ST}}^{*}=\tau_{\mathrm{ST}} \cdot \bar{n}_{\mathrm{e}, \kappa_{\max }} / \bar{n}_{\mathrm{e}}$, removes the hysteresis, as can be seen in figure $1(\mathrm{~d})$. The density corrected sawtooth period clearly decreases with increasing elongation. Interestingly most of the change in $\tau_{\mathrm{ST}}$ occurs in a relatively small interval in $\kappa$ from 1.9 to 2.0. The dependence of the sawtooth period on elongation is qualitatively consistent with a previous systematic scan of the elongation [5, 11], where increasing $\kappa$ from 1.1 to 2.1 reduced the sawtooth period by $30 \%$. The experiments, presented here, extend the previous results up to values of $\kappa \approx 2.6$, where the sawteeth 


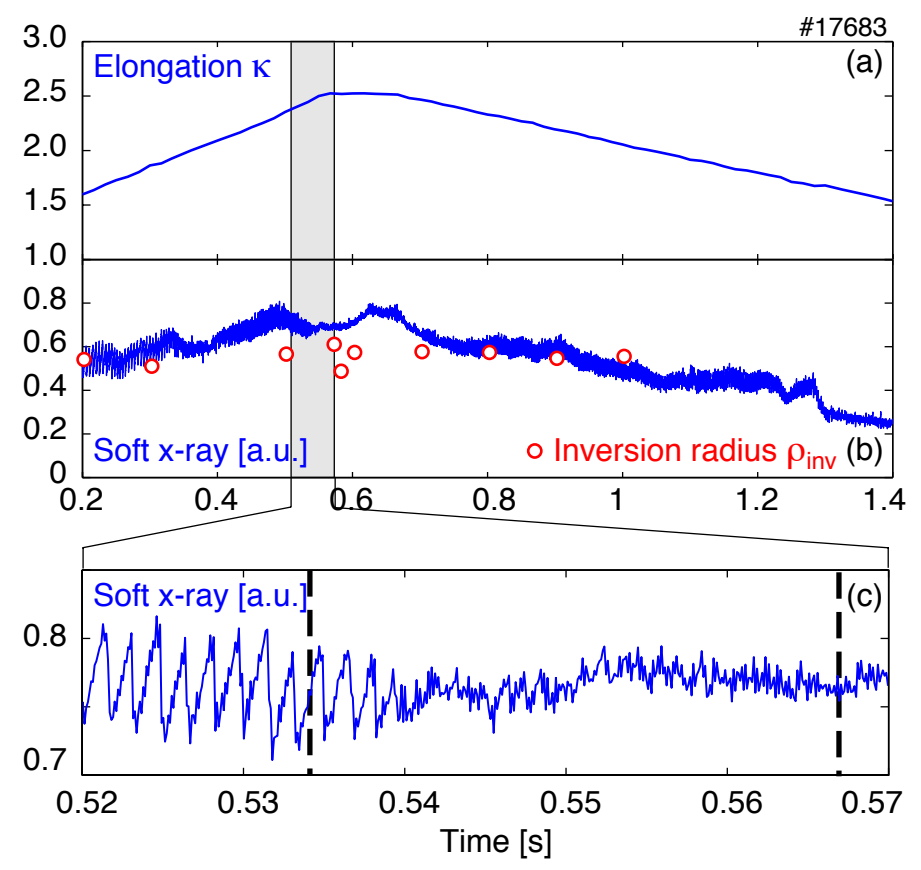

Figure 2. During the elongation ramp (a) in discharge \#17683 a central soft x-ray signal (b) shows the modification and (c) abrupt disappearance of sawteeth. The sawtooth inversion radius (b) does not change significantly before and after the nonsawtoothing phase. The dashed bars in (c) indicate the time-slices of the temperature profiles shown in figure 4 .

are seen to disappear.

The abrupt disappearance of sawteeth is a qualitatively new phenomenon. Figure 2 shows the sudden transition from a sawtoothing to a non-sawtoothing plasma in a similar discharge (\#17683). The last sawtooth has a period of typically $1-1.5 \mathrm{~ms}$ and an amplitude of $5 \%$ of the soft x-ray signal, which is well above the detection limit of the soft x-ray diagnostic. After this transition only sporadic sawteeth are observed. The sawteeth re-appear again at a similar value of $\kappa$ in the ramp-down of the discharge, as shown in figure $1(\mathrm{~d})$.

The sawtooth inversion radius $\rho_{\text {inv }}$ does not vary significantly before and after the non-sawtoothing phase. A measurement of the sawtooth inversion radius is obtained from a Singular Value Decomposition (SVD) of the reconstructed soft x-ray emissivity [12]. The inversion surface is mapped onto a flux surface, yielding $\rho_{\text {inv }}$. Here the flux surface is labeled by its poloidal flux coordinate $\rho_{\text {pol }}=\sqrt{\left(\psi-\psi_{0}\right) /\left(\psi_{a}-\psi_{0}\right)}$, where $\psi_{0}$ and $\psi_{a}$ are the values of the poloidal flux in the plasma centre and at the edge, 


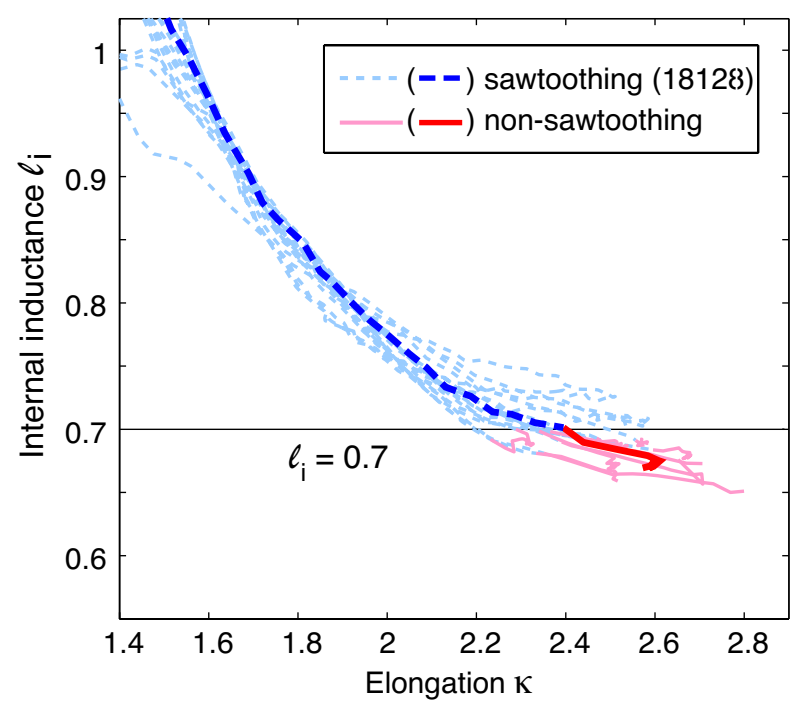

Figure 3. The transition from a sawtoothing to a non-sawtoothing phase in discharges with different densities and $I_{p}$-ramp up scenarios occurs at varying values of elongation, but at the same value of the internal inductance $\ell_{i}$. Discharge \#18128, for which a detailed analysis of its MHD activity is presented in section 2.3, is highlighted.

respectively. In discharge \#17683 the sawtooth inversion radius varies from $\rho_{\mathrm{inv}}=0.55$ to 0.60 , figure $2(\mathrm{~b})$. The values before the disappearance are similar to the values after the re-appearance. Sporadic sawteeth in the otherwise non-sawtoothing phase yield the same large inversion radius throughout the non-sawtoothing phase. The measurement of the inversion radius, therefore, indicates that the $q=1$ surface, which is closely related to the inversion radius [13], does not shrink prior to the transition.

The value of $\kappa$ where sawteeth disappear depends on the current profile. Figure 3 shows the evolution of the internal inductance $\ell_{i}$ as a function of $\kappa$ for several discharges. Both parameters are obtained from the equilibrium reconstruction code LIUQE [14]. The reconstructions are solely based on external magnetic measurements and, therefore, details of the current profile are not well known. While this causes an uncertainty in the value of $\ell_{i}$, the error is expected to be mostly systematic. The discharges differ in their density and $I_{p}$ ramp-up rates, leading to variations of the current profiles. The trajectories in the $\ell_{i}-\kappa$ diagram (figure 3 ) show that the transition from the sawtoothing to the non-sawtoothing phase occurs at about the same value of the internal inductance of $\ell_{i} \approx 0.7$, but at varying values of $\kappa$. 


\subsection{Electron temperature and density profiles}

The electron temperature $T_{\mathrm{e}}$ and electron density $n_{\mathrm{e}}$ profiles in the sawtoothing phase of discharge \#17683, shown in figure 2, are compared to the profiles in its non-sawtoothing phase, figure 4 . The profile measurements are obtained from a multi-chord Thomson scattering diagnostic. The small but rapid sawteeth at high elongation prevent a peaking of $T_{\mathrm{e}}$ in the core region of the plasma, figure $4(\mathrm{a})$, consistent with previous observations of sawteeth increasingly limiting the central pressure gradients towards high $\kappa$ [5]. The profile in the centre is flat up to mid-radius, even though the profile is measured halfway through the sawtooth ramp. The radial location of the roll-over in the $T_{\mathrm{e}}$ profile is close to the measured sawtooth inversion radius of $\rho_{\mathrm{inv}}=0.6$. Since the rapid sawteeth have a large effect on the central $T_{\mathrm{e}}$ and $n_{\mathrm{e}}$ profiles, it is surprising that the profiles do not vary significantly through the transition. In spite of the absence of sawteeth, the profile remains flat in the plasma core, figure 4(b). The position of the roll-over does not change either. Similarly, the electron density profiles before, figure 4(c), and after the transition, figure $4(\mathrm{~d})$, are flat in the plasma centre. The electron temperature and density profiles in the non-sawtoothing phase show that there must be another mechanism such as another MHD instability, which causes the increased radial transport and prevents the build-up of pressure gradients in the plasma core.

\subsection{MHD activity}

A detailed analysis of the MHD activity, typically observed in highly elongated plasmas, has been carried out for the discharge \#18128. In this discharge the sawteeth disappear at $t=0.50 \mathrm{~s}$ at an elongation of $\kappa=2.39$ and an internal inductance $\ell_{i}=0.70$. The discharge reaches a maximum elongation of $\kappa=2.65$ and terminates with a disruption at $t=0.67 \mathrm{~s}$. The trajectory of discharge \#18128 in the $\ell_{i}-\kappa$-plane is highlighted in bold in figure 3 .

Magnetic fluctuations $\delta B$ are measured with poloidal field probes installed inside the vacuum vessel. The probes are arranged in poloidal and toroidal arrays and can resolve low to medium poloidal and toroidal mode numbers $m$ and $n$. The toroidal array on the outboard mid-plane consists of 16 equidistantly spaced probes and resolves the toroidal mode numbers $n=0, \ldots 8$. The spectrograms of the $n=1$, 2, 3 mode components in discharge \#18128 are shown in figure 5 reveal a variety 

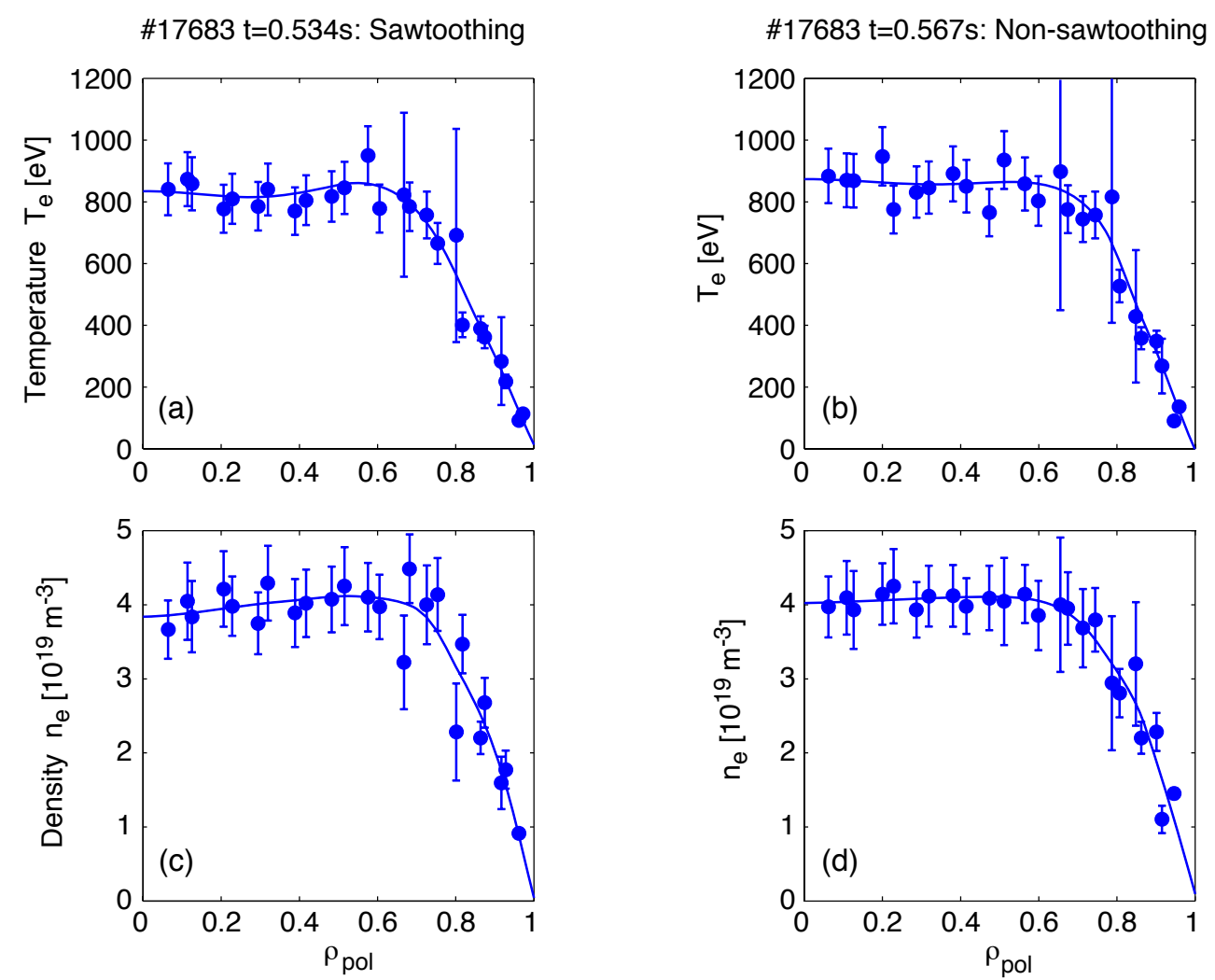

Figure 4. Electron temperature $(\mathrm{a}, \mathrm{b})$ and electron density $(\mathrm{c}, \mathrm{d})$ profiles measured by Thomson scattering before $(\mathrm{a}, \mathrm{c})$ and after $(\mathrm{b}, \mathrm{d})$ the transition from a sawtoothing to a non-sawtoothing phase in discharge \#17683 shown in figure 2(c).

of coherent oscillations. The poloidal mode number can be obtained from magnetic measurements with 38 poloidally distributed pick-up coils, which surround the whole poloidal circumference as indicated in figure 6(a). The experimental mode structure is retrieved using standard Fourier techniques or a singular value decomposition (SVD) of the full set of magnetic measurements. The interpretation of the poloidal mode structure in terms of poloidal mode numbers $m$ is complicated by the high elongation of the vacuum vessel and the great flexibility in plasma shape, which render a simple Fourier decomposition in the poloidal angle meaningless. The interpretation of the measured mode structure requires an inversion. The method used is based on a model of force- and divergence-free perturbation currents along the equilibrium field lines on a resonant surface [15]. A Biot-Savart integration, using the modeled perturbation current distribution, allows the calculation of the eddy currents in the vacuum vessel and, subsequently, also the magnetic field at the location of the magnetic probes. A comparison of phase and amplitude of the modeled and measured mode structure then 


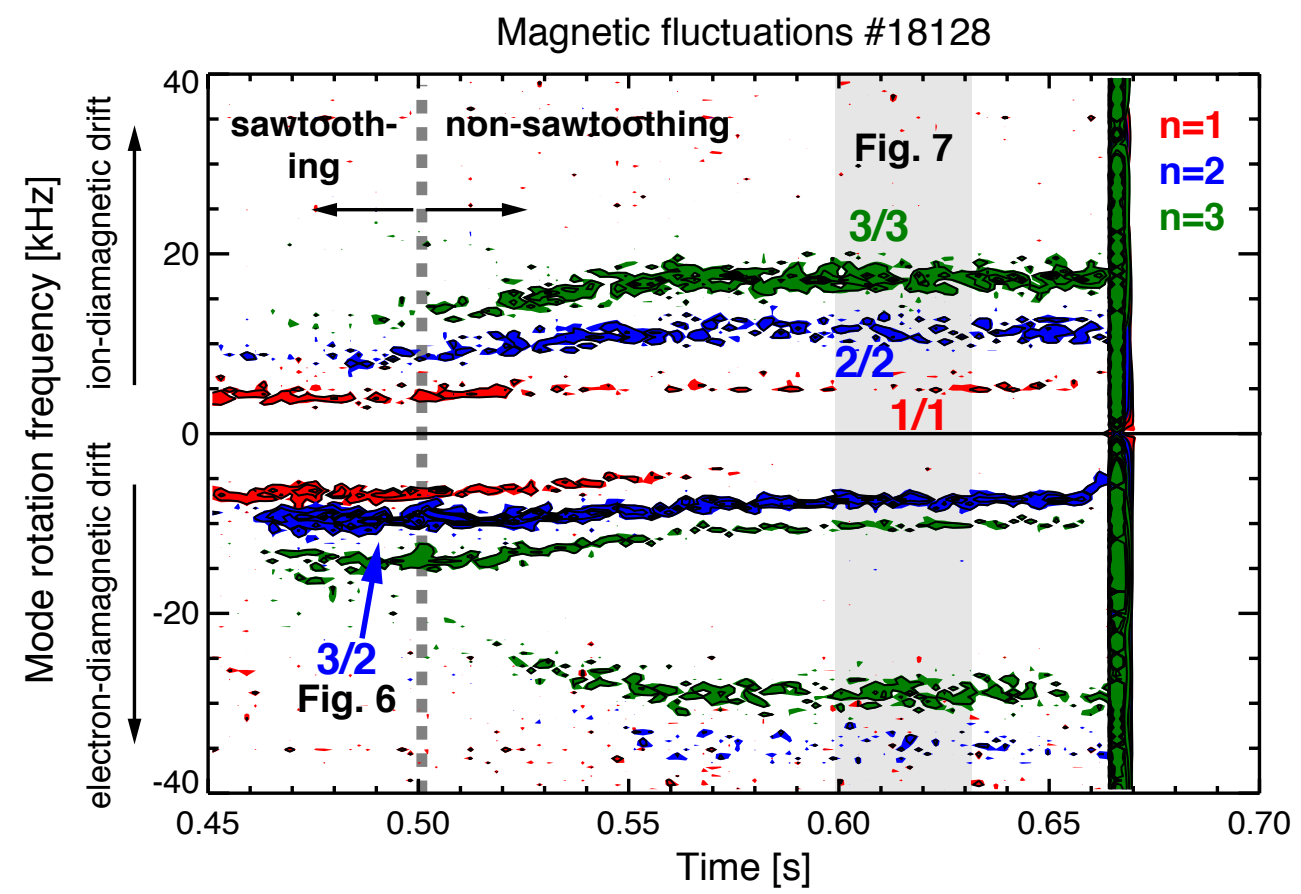

Figure 5. Spectrograms of the toroidal mode components $n=1$ (red), 2 (blue) and 3 (green) of magnetic fluctuation measurements in discharge \#18128. Positive frequencies correspond to a mode rotation in the direction of the ion diamagnetic drift. The sawteeth disappear at $t=0.50 \mathrm{~s}$ when $\kappa$ exceeds a value of 2.39 and $\ell_{i}$ drops below 0.70 as shown in figure 3 .

identifies the mode [16].

The discharge \#18128 shows the typical MHD activity encountered in highly elongated plasmas:

- The $m / n=3 / 2$ and $4 / 3$ modes are frequently observed in highly elongated plasmas [9]. They always have a single helicity and are thought to be tearing modes. Figure 5 shows an $n=2$ mode which starts to grow at $t=0.46 \mathrm{~s}$ and quickly saturates. It rotates with approximately $10 \mathrm{kHz}$ in the direction of the electron diamagnetic drift (negative frequencies in figure 5). The corresponding poloidal mode structure is obtained from the Fourier coefficients of the magnetic measurements at the mode frequency $\left|f_{n=2}\right|=10 \mathrm{kHz}$, shown in figure $6(\mathrm{~b}, \mathrm{c})$. The comparison of the measured poloidal mode structure with a modeled $m / n=3 / 2$ mode, fitting phase and amplitude, results in good agreement. As in \#18128, its appearance usually does not coincide with the disappearance of sawteeth.

- Disruptions at the ideal MHD $\beta$-limit at high elongation $(\kappa>2.4)$ and high 
(a)

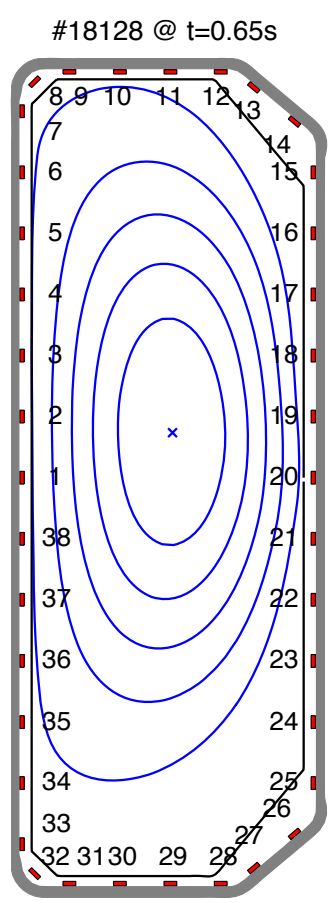

(b)

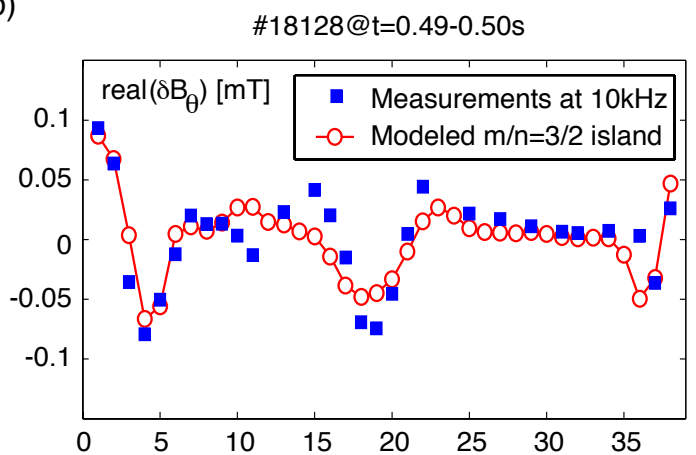

(c)

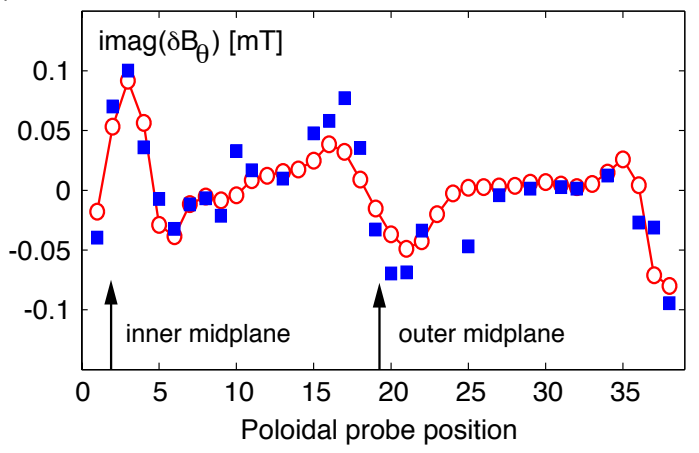

Figure 6. (a): Poloidal cross section of a highly elongated plasma $(\kappa=2.65)$ in the TCV vessel. The locations of 38 poloidally distributed magnetic pick-up coils are indicated. Real (b) and imaginary (c) part of the experimental mode structure, obtained from the Fourier coefficients at $10 \mathrm{kHz}$ of magnetic fluctuation measurements with the poloidal array (filled squares), and the modeled mode structure of an $m / n=3 / 2$ island (open circles) are in good agreement.

normalised current $\left(I_{N} \approx 3.5 \mathrm{MA} /(\mathrm{Tm})\right)$, which is strongly reduced with respect to the linear $\beta$-limit scaling with $I_{N}$, are preceded by an $n=1$ mode [9]. The precursor grows locked on a typical time scale of $\tau \approx 1 \mathrm{~ms}$. Its poloidal mode structure is dominantly $m=2$. Such a precursor is also seen in \#18128 at $0.67 \mathrm{~s}$ but does not appear in the magnetic fluctuation spectrogram because it does not rotate.

The disappearance of sawteeth at $t=0.50 \mathrm{~s}$, however, coincides with the onset of new type of MHD activity. The Fourier spectrum of the toroidal mode components (figure 5) shows that the emergence of simultaneous $n=2$ and 3 modes when sawteeth disappear. These modes rotate with approximately 10 and $17 \mathrm{kHz}$, respectively, in the ion diamagnetic drift direction (positive frequencies in figure 5). While the signal amplitude of the poloidal probe array is too low for a mode identification, the poloidal structure of modes in the plasma core can be resolved using the tomographic 


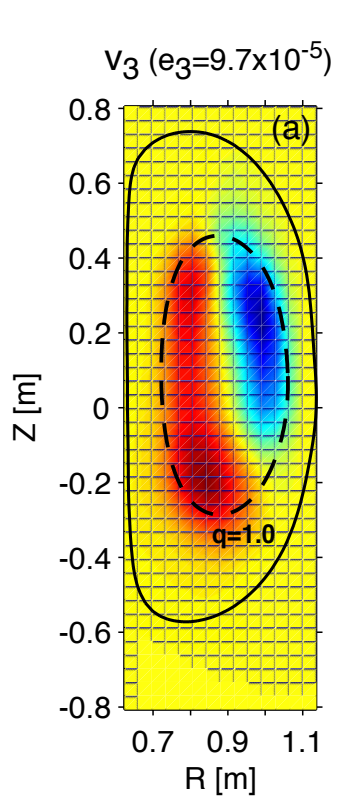

Soft x-ray tomography: \#18128 - $\mathrm{t}=0.600-0.6325 \mathrm{~s}$
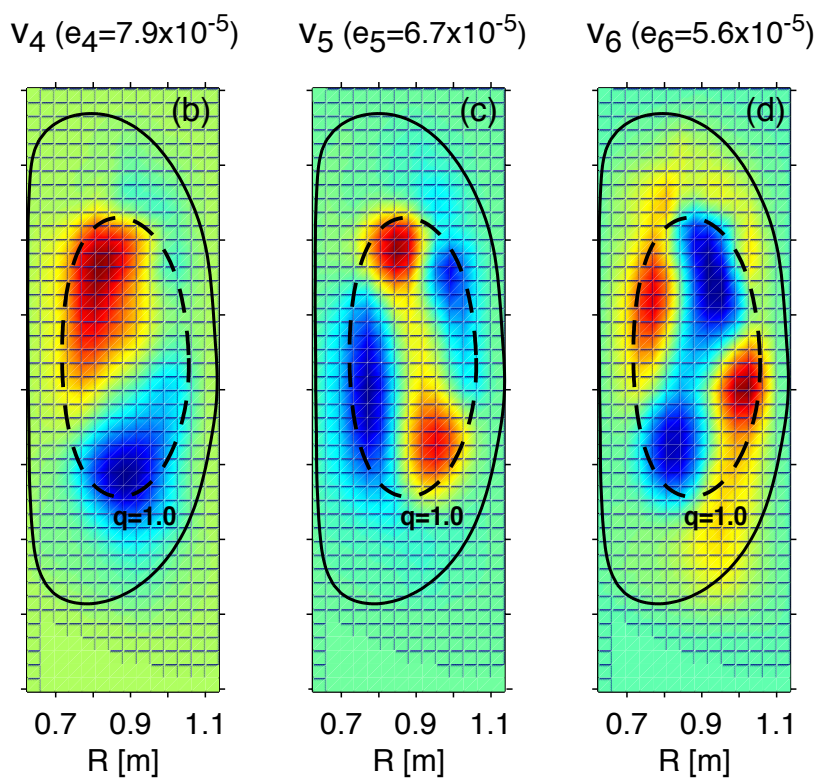

$\mathrm{v}_{7}\left(\mathrm{e}_{7}=4.2 \times 10^{-5}\right)$
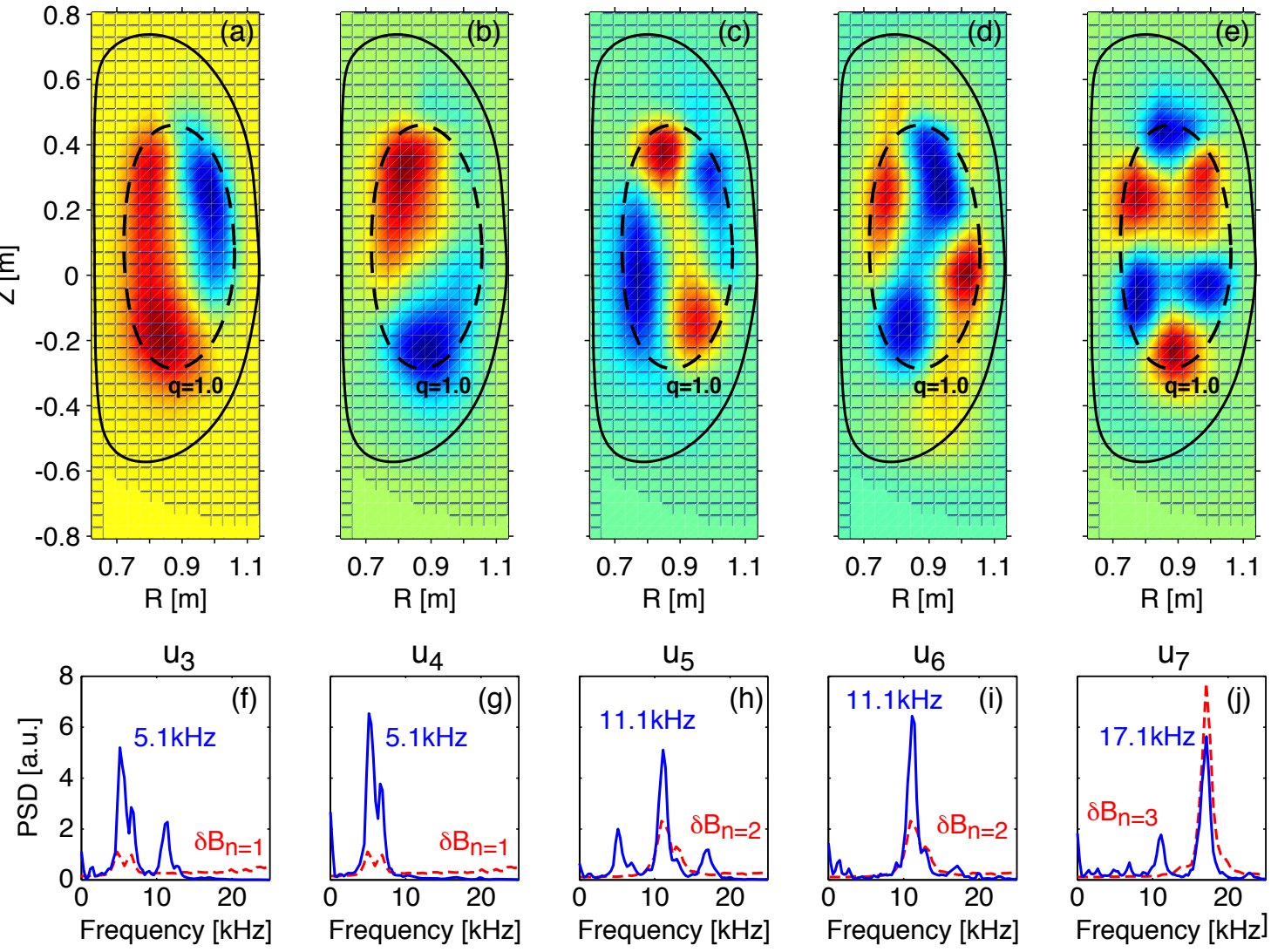

Figure 7. Third through seventh spatial eigenvectors (topo) (a-e) and power spectral densities (PSD) of the corresponding temporal eigenvectors (chrono) ( $f-j$ ) of the singular value decomposition of the tomographically reconstructed soft x-ray emissivity in a non-sawtoothing phase of discharge \#18128. The 3rd and 4th topos (a,b) and corresponding chronos (f,g) reveal a $m=1$ mode rotating at $5.1 \mathrm{kHz}$ and the 5 th and 6 th topos (c,d) and chronos (h,i) a $m=2$ mode at $11.1 \mathrm{kHz}$. The 7 th topo (e) and chrono (j) shows a $m=3$ structure oscillating with $17.1 \mathrm{kHz}$. A comparison with the (positive) frequencies of the toroidal mode component of magnetic measurements, figure 5 , in the same time interval ( $\mathrm{f}-\mathrm{j}$, dashed) identifies the $1 / 1,2 / 2$ and $3 / 3$ mode.

reconstruction of the soft x-ray emissivity. The soft x-ray tomography diagnostic on TCV [17] uses soft x-ray emission measurements along 200 lines-of-sight which are presently sampled with a frequency of up to $77 \mathrm{kHz}$.

An SVD analysis of the reconstructed emissivity in the non-sawtoothing interval from $t=0.60$ to $0.6325 \mathrm{~s}$ in discharge \#18128, shown in figure 7 , reveals coherent modes. The spatial (topo) and temporal (chrono) eigenvector pairs $\left(v_{k}, u_{k}\right)$ are ordered 
according to their contribution $e_{k}$ to the total signal energy. The largest contributions $\left(e_{1,2}>99.9 \%\right)$ come from the mean emissivity contours and from elongation changes within the analysed interval (first and second singular value), which are not shown here. The third and fourth topos $v_{3}$ and $v_{4}$, figure $7(\mathrm{a}, \mathrm{b})$, clearly show a rotating $m=1$ mode. The power spectral densities of the corresponding chronos $u_{3}$ and $u_{4}$, figure $7(\mathrm{f}, \mathrm{g})$, yield a peak at $5.1 \mathrm{kHz}$ which is the same frequency as the low amplitude $n=1$ mode in the ion diamagnetic drift direction seen with the magnetic fluctuation measurements. Figure $7(\mathrm{a}-\mathrm{e})$ shows also the $q=1$ surface as determined by a LIUQE equilibrium reconstruction. The direct identification of a resonance location from the topos is, however, obstructed by the dependence of the mode displacement on the gradients of the mean emissivity profile. The fifth and sixth topos $v_{5}$ and $v_{6}$, figure $7(\mathrm{c}, \mathrm{d})$, show a $m=2$ mode, which rotates with $11.1 \mathrm{kHz}$ figure $7(\mathrm{~h}, \mathrm{i})$. This frequency matches the frequency of the $n=2$ mode in the ion diamagnetic drift direction. The signal energy of the two topo/chrono pairs $e_{5,6}$ is about $30 \%$ lower than the signal energy of the $1 / 1$ mode. The seventh topo $v_{7}$, figure $7(\mathrm{e})$, has a clear $m=3$ structure. The peak in the PSD of the corresponding chrono $u_{7}$, figure $7(\mathrm{j})$, at $17.1 \mathrm{kHz}$ matches the frequency of the $n=3$ mode. A similar structure and frequency is seen in the ninth topo/chrono, but they are not as clear. The resolution of the $m=3$ structure could be hampered by the presence of a second, low amplitude $m=3$ mode on the $q=3 / 2$ surface, which is seen in the magnetic measurements. The proximity of the $3 / 2$ surface to the plasma edge and, hence, to the magnetic probes increases the amplitude of the magnetic fluctuation measurements, but leads to a low soft x-ray emissivity. Assuming that the seventh eigenvector pair corresponds only to the $3 / 3$ mode, its signal energy is $50 \%$ lower than the signal energy of the $1 / 1$ mode.

The combination of magnetic fluctuation and soft x-ray measurements reveals the structure of the MHD activity related to the disappearance of sawteeth. The typical sawtooth cycles are replaced by continuous oscillations. These oscillations are resonant on the $q=1$ surface and in addition to the typical sawtooth related $1 / 1$ activity show strong higher harmonics, namely the $2 / 2$ and $3 / 3$ modes. These modes rotate in the ion diamagnetic direction. However, it is not known how this relates to the bulk plasma rotation which has not been measured in these discharges. The amplitudes $\xi_{m / n}$ of these modes, which can be estimated by the square root of the signal energy of the 
corresponding eigenvectors of the soft x-ray tomography, are approximately related by the ratio of $\xi_{1 / 1}: \xi_{2 / 2}: \xi_{3 / 3}=1: 0.85: 0.7$. The higher mode numbers are, therefore, much more important than during a normal sawtooth crash [18]. Saturated core MHD activity with a slowly decreasing $m$ spectrum has also been observed in high $\kappa$ discharges, where off-axis electron cyclotron heating broadens the current profile [19].

\section{MHD stability}

Previous experiments have shown the decrease of sawtooth stability with elongation and linked the sawtooth crash at high $\kappa$ and/or low $\delta$ to the stability of the ideal internal kink mode [5]. For $\kappa>1.6$, the maximum pressure gradient on the $q=1$ surface decreases continuously with increasing $\kappa$. This is in qualitative agreement with an analytic prediction of the leading order elongation effect on the growth rate of the internal kink mode [20,21]. Assuming that $\Delta q=1-q_{0}$ is small, which is compatible with the low values of $\ell_{i}$ in the highly elongated TCV plasmas, the analytic prediction for the growth rate $\gamma_{\text {ana }} \tau_{\mathrm{A}} \propto \epsilon_{1}^{2}\left(\kappa_{1}-1\right) \beta_{p 1} s_{1}^{-1}$ increases with elongation. Here, $\tau_{\mathrm{A}}$ is the toroidal Alfvén time, $s$ the magnetic shear, $\epsilon$ the inverse aspect ratio, $\beta_{p 1}=\left(\langle p\rangle_{1}-p_{1}\right) /\left(B_{p 1}^{2} / 2 \mu_{0}\right)$ the Bussac poloidal beta [22] with $p$ being the plasma pressure and $B_{p}$ the poloidal magnetic field. The index 1 denotes the quantities at the $q=1$ surface. Moreover, the analytic calculations predict a critical elongation at the $q=1$ surface $\kappa_{1}^{c}$, above which the ideal internal kink mode is unstable even for $\beta_{p 1}=0$, which corresponds to a flat central pressure profile. Taking into account higher order terms and assuming parabolic profiles and $\Delta q=0.05$ the analytic expansion in [20] yields $\kappa_{1}^{c} \approx 1.54$, which is significantly lower than values of $\kappa_{1} \approx 2$ typically observed at the transition in the TCV discharges. It is, however, expected that the growth rate of the ideal internal kink mode has to exceed a fraction of the ion diamagnetic frequency $\omega_{* i}$ to overcome stabilizing diamagnetic effects in order for the mode to become unstable [23]. A more complete discussion of shaping effects on $\gamma_{\text {ana }}$ can be found in reference [21].

The growth rate of the ideal MHD internal kink mode $\gamma_{\mathrm{KINX}}$ is calculated using the KINX code [24]. In these calculations a fixed boundary is assumed, which leads to systematically lower growth rates than the free-boundary case. Figure 8 shows the evolution of $\gamma_{\mathrm{KINX}} \tau_{\mathrm{A}}$ in several high- $\kappa$ discharges. The sawteeth disappear when $\gamma_{\mathrm{KINX}} \tau_{\mathrm{A}}$ attains the highest values. The range of $\gamma_{\mathrm{KINX}} \tau_{\mathrm{A}}$ from 0.045 to 0.060 , where discharges 


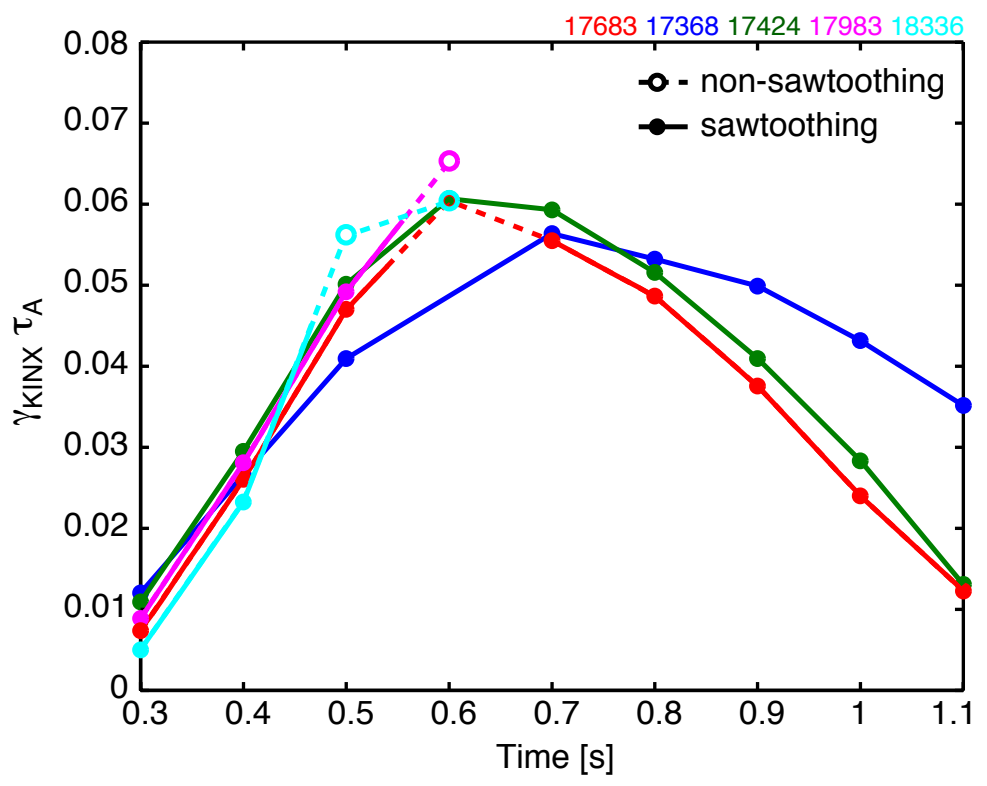

Figure 8. Evolution of the growth rate of the ideal internal kink mode calculated with the KINX code $\gamma_{\mathrm{KINX}}$, in several high- $\kappa$ discharges. Intervals, where sawteeth disappear, are indicated (dashed line, open symbols).

are found sawtoothing and non-sawtoothing, can be caused by uncertainties in the equilibrium reconstructions and variations in $\omega_{* i}$, which is not directly measured.

In order to verify parametric dependencies and to develop a simple formula for sawtooth modeling $[23,25]$, functional fits of $\gamma_{\mathrm{KINX}} \tau_{\mathrm{A}}$ calculated with KINX for a large set of equilibria have been proposed [21],

$$
\begin{aligned}
& \gamma^{\mathrm{fit} 1} \tau_{\mathrm{A}}=0.45 \frac{\epsilon_{1} \kappa_{1}}{1+7 \epsilon_{1} s_{1}}\left(\beta_{p 1}-\beta_{p 1}^{c, \mathrm{fit} 1}\right), \\
& \beta_{p 1}^{c, \text { fit } 1}=0.7-0.5 \kappa_{1} .
\end{aligned}
$$

While the fit $(1 \mathrm{a}, \mathrm{b})$ has been derived from the entire set of equilibria, a modification especially for high $\kappa$ and low $\ell_{i}$ has been proposed [21],

$$
\begin{aligned}
& \gamma^{\text {fit } 2} \tau_{\mathrm{A}}=0.44 \frac{\epsilon_{1} \kappa_{1}}{1+7 \epsilon_{1} s_{1}}\left(\beta_{p 1}-\beta_{p 1}^{c, \text { fit } 2}\right), \\
& \beta_{p 1}^{c, \text { fit } 2}=0.9-\left(0.6+0.1 s_{1}\right) \kappa_{1} .
\end{aligned}
$$

The values of $\gamma^{\text {fit }}$ obtained from both fits for the discharges shown in figure 8 are compared to the KINX calculation. Figure 9 shows that both fits reproduce the trend of the numerical calculation. For $\kappa>2.2$ the second fit $(2 \mathrm{a}, \mathrm{b})$ leads to a remarkably good agreement with the KINX calculations. Both parametric fits, (1a,b) and (2a,b), clearly show the destabilizing effect of elongation with $\beta_{p 1}^{c}$ decreasing with increasing $\kappa_{1}$ 


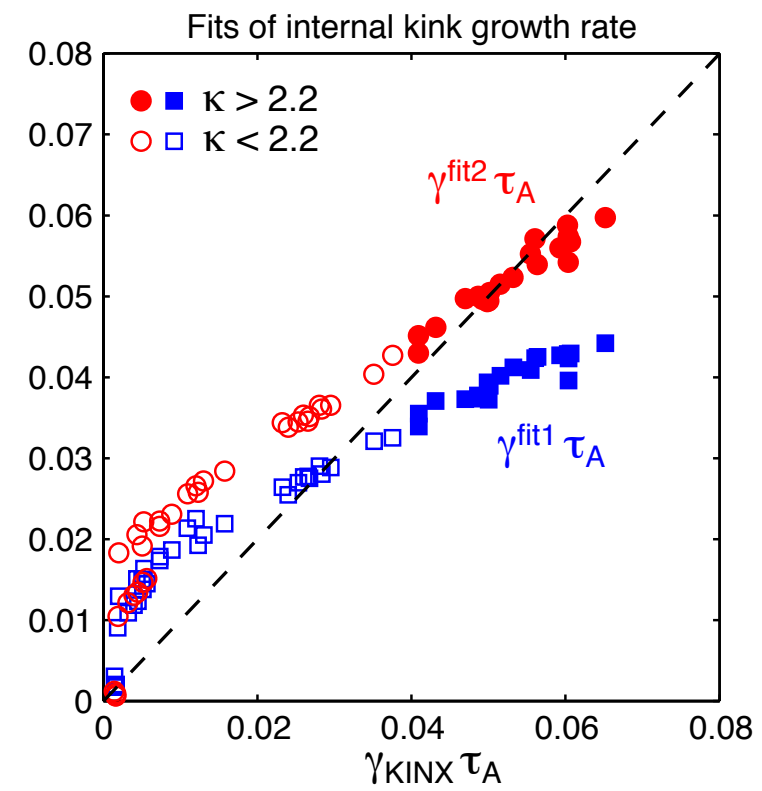

Figure 9. Comparison of the parametric fits of the ideal internal kink growth rate $\gamma^{\text {fit1 }}(1 \mathrm{a}, \mathrm{b})$ and $\gamma^{\text {fit2 }}(2 \mathrm{a}, \mathrm{b})$ with KINX calculations of $\gamma_{\mathrm{KINX}}$. At high $\kappa$ (filled symbols: $\kappa>2.2) \gamma^{\text {fit2 }}$ is a good estimate of $\gamma_{\mathrm{KINX}}$.

and $\gamma \tau_{\mathrm{A}}$ increasing with $\kappa_{1}$ once $\beta_{p 1}>\beta_{p 1}^{c}$. The parametric fits also show the sensitivity to the current profile. Although $\ell_{i}$ is not used as a fit parameter, changes in $\ell_{i}$ lead to changes in the penetration of magnetic shear and plasma shaping inside the plasma and, therefore, alters $s_{1}$ and $\kappa_{1}$.

It is, therefore, likely that the elongation in the experiment is sufficiently high so that the ideal internal kink overcomes stabilising diamagnetic effects and prevents any build-up of pressure gradients on the $q=1$ surface and, hence, sawtooth crashes. It is possible that a resistive mode located at the $q=1$ surface becomes unstable as the plasma approaches the ideal stability limit. The onset of resistive modes in the vicinity of ideal stability limits is routinely observed at higher rational surfaces [26]. The onset and subsequent saturation of such a resistive mode prior to the ideal limit is a possible explanation of the abrupt transition from a finite sawtooth amplitude to a continuous mode in the non-sawtoothing phase. Saturated resistive $m / n=1 / 1$ modes at the $q=1$ surface have also been observed in recent JET hybrid discharges, albeit at lower elongation and with a significant contribution from non-inductive current drive [27]. Interestingly, the JET observations suggest that low $\ell_{i}$ is beneficial for the mode saturation. The saturation of the core MHD activity in TCV should be studied with a 
3-D nonlinear MHD code, but is out of the scope of this article.

\section{Summary}

The unique design of the TCV tokamak has allowed studies of the stability of extremely elongated plasmas. The axisymmetric mode and the ideal kink mode impose well known lower and upper current limits, respectively. Occasionally, the $m / n=3 / 2$ island, rotating in the direction of the electron diamagnetic drift, is observed. Previous studies of the stability of sawteeth in ohmically heated plasmas, which showed a decrease of the sawtooth period and amplitude with increasing elongations, despite a constant inversion radius, have been verified up to $\kappa=2.6$. At a sufficient elongation, sawteeth are observed to disappear abruptly. The value of $\kappa$ at the transition depends on the current profile, notably $\ell_{i}$ and varies from $\kappa=2.2$ to 2.6 . The electron density and temperature profiles in the plasma centre remain flat throughout the transition. The analysis of magnetic fluctuation measurements and the soft x-ray tomography has shown that the sawteeth are replaced by continuous $m / n=1 / 1,2 / 2$ and $3 / 3$ modes, which are resonant on the $q=1$ surface and seem to prevent the peaking of the profiles. The amplitudes of the $2 / 2$ and $3 / 3$ modes are of the same order of magnitude as the $1 / 1$ mode. Analytic and numerical ideal MHD calculations show that at a sufficiently high elongation the internal kink mode becomes unstable even with a flat pressure profile inside $q=1$. The disappearance of sawteeth in TCV is well correlated with the ideal kink growth rate exceeding a positive threshold value. It is suggested that the continuous MHD activity, which replaces sawteeth, are resistive modes destabilised in the proximity of the ideal MHD limit. Such a transition can explain the abrupt disappearance of finite amplitude sawteeth at high elongation.

\section{Acknowledgments}

This work was partly funded by the Fonds National Suisse de la Recherche Scientifique. Contributions of R. Duquerroy to the analysis of the soft x-ray measurements are gratefully acknowledged. 


\section{References}

[1] Troyon F et al 1984 Plasma Phys. Control. Fusion 26209

[2] ITER Physics Expert Groups on Confinement and Transport and Confinement Modelling and Database, ITER Physics Basic Editors, 1999 Nucl. Fusion 392175

[3] Weisen H et al 1998 Plasma Phys. Control. Fusion 401803

[4] Sauter O et al 2002 Phys. Rev. Lett. 88105001

[5] Reimerdes H, Pochelon A, Sauter O, Goodman T P, Henderson M A and Martynov An 2000 Plasma Phys. Control. Fusion 42629

[6] Weisen $\mathrm{H}$ et al 2002 Nucl. Fusion 42136

[7] Lazarus E A, Waelbroeck F L, Luce T C et al 2006 Plasma Phys. Control. Fusion 48 L65

[8] Hofmann F et al 2001 Plasma Phys. Control. Fusion 43 A161

[9] Hofmann F, Sauter O, Reimerdes H, Furno I and Pochelon A 1998 Phys. Rev. Lett. 812918

[10] Campbell D J, Gill R D, Gowers C W, Wesson J A et al 1986 Nucl. Fusion 261085

[11] Pochelon A et al 2001 Nucl. Fusion 411663

[12] Furno I et al 2001 Nucl. Fusion 41403

[13] Pietrzyk Z A et al 1999 Nucl. Fusion 39587

[14] Hofmann F and Tonetti G 1988 Nucl. Fusion 281871

[15] Schittenhelm M, Zohm H and ASDEX Upgrade team 1997 Nucl. Fusion 371255

[16] Reimerdes H 2001 Ph.D. thesis, EPFL thesis nb. 2399 and Lausanne report LRP 700/01

[17] Anton M et al 1996 Plasma Phys. Control. Fusion 381849

[18] Duperrex P A, Pochelon A, Edwards A W and Snipes J A 1992 Nucl. Fusion 321161

[19] Pochelon A, Arnoux G, Camenen Y, Scarabosio A et al 2002 Proc. 19th IAEA Fusion Energy Conf., Lyon, France, 14-19 October 2002 (Vienna: IAEA) IAEA-CN-94/EX/P5-14 and Scarabosio A 2006 Ph.D. thesis, EPFL thesis nb. 3609

[20] Eriksson H G and Wahlberg C 2002 Phys. Plasmas 91606

[21] Martynov An, Graves J P and Sauter O 2005 Plasma Phys. Control. Fusion 471743

[22] Bussac M N, Pellat R, Edery D and Soulé J L 1975 Phys. Rev. Lett. 351638

[23] Porcelli F, Boucher D and Rosenbluth M N 1996 Plasma Phys. Control. Fusion 382163

[24] Degtyarev L et al 1997 Comput. Phys. Commun. 10310

[25] Graves J P et al 2005 Plasma Phys. Control. Fusion 47 B121

[26] Brennan D P, Strait E J, Turnbull A D et al 2002 Phys. Plasmas 92998

[27] Buratti P et al 2005 Proc. 32nd EPS Conf. on Plasma Physics and Controlled Fusion, Tarragona, Spain, 27 June - 1 July, 2005) (ECA) vol 29C O-1.004 